\title{
Extending the Syntax and Semantics of the Hybrid Functional-Object-Oriented Scripting Language FOBS with FEDELE
}

\author{
James Gil de Lamadrid \\ Bowie State University/Computer Science Department, Bowie, Maryland, 20715, United States of America \\ E-mail: jgildelamadrid@bowiestae.edu
}

\begin{abstract}
We describe the programming language FOBS-X (Extensible FOBS). FOBS-X is interpreted, and is intended as a universal scripting language. One of the more interesting features of FOBS-X is its ability to be extended, allowing it to be adopted to new scripting environments. FOBS-x is structured as a core language that is parsed by the interpreter, and an extended language that is translated to the core by macro expansion. The syntax of the language can easily be modified by writing new macros. The library for FOBS-X is reconfigurable, allowing the semantics of the language to be modified, and adapted to facilitate the interaction with interfaces to new scripting environments. This paper focuses on the tools used for the semantic extension of the language. A tool called FEDELE has been developed, allowing the user to add library modules to the FOBS-X library. In this way the semantics of the language can be enhanced, and the language can be adapted.
\end{abstract}

Index Terms -Functional, object-oriented, programming language.

\section{INTRODUCTION}

Tools and techniques from both the object-oriented and the functional paradigms are valuable to the programmer. Techniques from the functional paradigm provide elegant solutions to many problems. Many other problems are best solved using the concept of communicating objects inherent in the object oriented paradigm. FOBS-X is a single language that offers the user the expressive power of both paradigms, allowing the user a choice of tools when analyzing a problem, but requiring only fluency in one language. The language FOBS-X is a version of the language FOBS, described by Gil de Lamadrid \& Zimmerman ${ }^{[4]}$. The changes to FOBS involve simplification of the pointers used in scoping rules.

FOBS-X shares many characteristics with functional languages. In particular, it is characterized by the following features:

- A single data type called a FOB, that is a simple and elegant structure that functions as both a function and an object.

- Stateless programming. Mutable objects do not exist in the FOBS-X runtime environment. Instead, mutation is simulated by creating new objects that incorporate the required changes.

- A simple form of inheritance. A sub-FOB can be built by combining a new FOB with a super-FOB. The sub-FOB inherits all attributes from the superFOB in the process.

- Scoping rules that support attribute overriding in inheritance. This enables a sub-FOB to modify or replace behaviors and attributes of a super-FOB.

- The ability to modify syntax through a macro expansion capability.

- A tool for easily writing new library modules, allowing the semantics of FOBS-X to be modified to fit differing scripting requirements.

Many scripting languages are weakly typed. FOBS follows this trend. Often the justification for weak typing is that it relieves the programmer from the burden of strict type enforcement. However, it also results in a situation in which type errors are not detected until late. The justification for weak typing in FOBS-X is based on two points. The first is that FOBS-X only has one data type, making strong type checking, based on syntax, almost impossible. The second point is that with interpreted languages the distinction between parsing and execution is much more blurred than with compiled languages, and so type checking before execution becomes much less important.

Several researchers have built hybrid language systems, in an attempt to combine the functional and objectoriented paradigms, but have sacrificed referential transparency in the process. A language called PROOF, developed by Yau et al. [11] attempts to incorporate objects into the functional paradigm. However, the modifications do little to take into account the functional programming style. Alexandrescu [1] presents the language $D$, which is a rework of the language C, transforming it into a more natural scripting language that is similar to Javascript and Ruby.

Scala by Odersky et al. ${ }^{[12]}$ is a language compiled to the Java Virtual Machine. The claim for Scala is that it implements a hybrid of the functional and object oriented paradigms, but, in fact, it tends toward the imperative language end of the spectrum. Scala is a class based language that is proposed as a tool to write web-servers. 
It is implemented as a small core language, along with a library that implements many of its capabilities. The same structure in FOBS allows the capabilities of the language to be easily extended.

The two languages FLC by Beaven et al. ${ }^{[2]}$, and FOOPS by Goguen and Mesegner [6] seek to preserve functional features. In FOOPS, functional features have been augmented by adding in support for ADTs. FLC, in our opinion, takes an approach that is conceptually simpler. In FLC, classes are represented as functions. FOBS is based on this same representation scheme. The class structure, however, has been removed from FOBS. The role of the class as a "factory" of individual objects, each with their own state, is not applicable in a stateless environment such as that in FOBS. A stateless system lends itself better to a prototype system, in which a single prototype object is copied with slight modifications to produce variants.

Another language that implements object-orientation while maintaining a mostly functional approach is OCAML $^{[8]}$. OCAML is built around ML, but has added elements enabling object-oriented and imperative programming. The creation of objects is supported by a record structure, and stateful programming is supported by mutable objects. The importance of mutation in object-orientation is discussed later in the paper. And, although important, we felt that mutation should be isolated and controlled. This helps preserve the overriding computation model of FOBS, which prominently features referential transparency. OCAML has a distinctly non-declarative nature, resulting from the tight integration of mutable objects into the computational model.

Scripting languages have tended to avoid the functional paradigm. Several object-oriented scripting languages such as Python [3] are available. Python is mostly object-oriented, although its support for functional programming is decent, including LISP like characteristics such as dynamic typing and anonymous functions. However, Python lacks referential transparency. We view this as one of the more significant features of FOBS. We also felt, when designing FOBS, that a simpler data structure could be used to implement objects and the inheritance concept, than was used in this popular language. FOBS combines functional programming and object-orientation into a single elegant hybrid language, offering both tools to the user. This is not done by adding in features from both paradigms, as do languages like Python or FOOPS, but rather by incorporating a single structure that embodies both paradigms, and unifies them.

\section{LANGUAGE DESCRIPTION}

FOBS-X is built around a core language, core-FOBS-X. Core-FOBS-X has only one type of data: the FOB. A simple FOB is a quadruplet,

$$
[\mathrm{m} \text { i } \rightarrow \text { e } \hat{\rho}]
$$

The FOB has two tasks. Its first task is to bind an identifier, $i$, to an expression, $e$. The e-expression is unevaluated until the identifier is accessed. Its second task is to supply a return value when invoked as a function. $\rho$ (the $\rho$-expression) is an unevaluated expression that is evaluated and returned upon invocation.

The FOB also includes a modifier, $m$. This modifier indicates the visibility of the identifier. The possible values are: "“+", indicating public access, " $\sim$, indicating protected access, and " $\$ "$, indicating argument access. Identifiers that are protected are visible only in the FOB, or any FOB inheriting from it. An argument identifier is one that will be used as a formal argument, when the FOB is invoked as a function. All argument identifiers are also accessible as public.

As an example, the FOB

$$
\left[{ }^{\prime}+\mathrm{x}->3{ }^{\wedge} 6\right]
$$

is a FOB that binds the variable $x$ to the value 3 . The variable $x$ is considered to be public, and if the FOB is used as a function, it will return the value 6 .

Primitive data is defined in the FOBS library. The types Boolean, Char, Real, and String have constants with forms close to their equivalent $C$ types. The Vector type is a container type, with constants of a form close to that of the Prolog list. For example, the vector

$$
\text { ["abc", 3, true] }
$$

represents an ordered list of a string, an integer, and a Boolean value. Semantically, a vector is more like the Java type of the same name. It can be accessed as a standard list, using the usual car, $c d r$, and cons operations, or as an array using indexes. It is implemented as a Perl list structure. Unlike the Java vector type, the FOBS-X vector type is immutable. The best approximation to the mutate operation is the creation of a brand new modified vector.

There are three operations that can be performed on any FOB. These are called access, invoke, and combine. An access operation accesses a variable inside a FOB, provided that the variable has been given a public or argument modifier. As an example, in the expression

$$
[`+x->3 \text { ' } 6] . x
$$

the operator "," indicates an access, and is followed by the identifier being accessed. The expression would evaluate to the value of $x$, which is 3 .

An invoke operation invokes a FOB as a function, and is indicated by writing two adjacent FOBs. The first FOB is the the invoked FOB, and the second FOB contains the actual arguments for the function invocation. In the following example

$$
[\nmid \$ \mathrm{y}->-\hat{y} \cdot+[1]][3]
$$

a FOB is defined that binds the variable $y$ to the empty 
FOB and returns the result of the expression $y+1$, when used as a function. When the example is used as a function by the invoke operation, since $y$ is an argument variable, the binding of the variable $y$ to the empty FOB is considered only a default binding. This binding is replaced by a binding to the actual argument, 3 . To do the addition, $y$ is accessed for the FOB bound to the identifier + , and this FOB is invoked with 1 as its actual argument. The result of the invocation is 4 .

In an invocation, it is assumed that the second operand is a vector. This explains why the second operand in the above example is enclosed in square braces. Invocation involves binding the actual argument to the argument variable in the FOB, and then evaluating the expression, giving the return value.

A combine operation is indicated with the operator ";". It is used to implement inheritance. In the following example

$$
\begin{aligned}
& \text { [ ' } \left.+\mathrm{x}->33^{\prime}\right] \text {; } \\
& {\left[' \$ y-\wedge_{-} \mathrm{x} .+[\mathrm{y}]\right]}
\end{aligned}
$$

two FOBs are combined. The super-FOB defines a public variable $x$. The sub-FOB defines an argument variable $y$, and a $\rho$-expression. Notice that the sub-FOB has unrestricted access to the super-FOB, and is allowed access to the variable $x$, whether modified as public, argument or protected.

The FOB resulting from Expression (1) can be accessed, invoked, or further combined. For example the code

$$
\begin{aligned}
& \left(\left[{ }^{\prime}+\mathrm{x}->3^{\wedge}\right]\right. \text {; } \\
& {\left[\left(\$ y-\hat{y}^{-} \mathrm{x} .+[\mathrm{y}]\right]\right) . \mathrm{x}}
\end{aligned}
$$

evaluates to 3 , and the code

$$
\begin{aligned}
& \left(\left[{ }^{‘}+\mathrm{x}->3{ }^{\wedge}\right]\right. \text {; } \\
& \left.\left.[' \$ \mathrm{y}-\rangle_{-} \mathrm{x}+[\mathrm{y}]\right]\right) \quad[5]
\end{aligned}
$$

evaluates to 8 .

Multiple combine operations result in FOB stacks, which are compound FOBs. For example, the following code creates a FOB with an attribute $x$ and a twoargument function that multiplies its arguments together. The code then uses the FOB to multiply 9 by 2 .

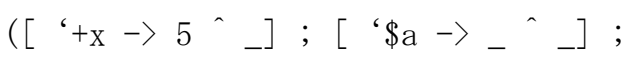

$$
\begin{aligned}
& \left.\left[\text { ' } \$ b->{ }_{-} \text {a. } \star[b]\right]\right)[9,2]
\end{aligned}
$$

In the invocation, the arguments are substituted in the order from top to bottom of the FOB stack, so that the formal argument $a$ would be bound to the actual argument 2, and the formal argument $b$ would be bound to 9 .

In addition to the three primitive FOBS operations, many operations on primitive data are defined in the FOBS library. These operations include the usual arithmetic, logic, and string manipulation operations. In addition, conversion functions provide conversion from one primitive type to another, when appropriate.

Example (2) presents a larger example to demonstrate how FOBS code might be used to solve more complex programming problems. In this example we define a FOB that implements a standard up-counter. The FOB structure is shown in Fig. 1, using UML. The outermost FOB implements the UML class called CounterMaker, that copies a prototype to create new counters. The counters are known as the class Counter in Fig. 1. CounterMaker creates a new Counter when its function makeCounter is called. The argument to makeCounter, val, becomes the initial value of the counter. The counter contains an instance variable, count, that contains the current count value. When the Counter FOB is invoked, the value of the variable count is returned. The counter also contains a function inc that "increments" the counter. Since FOBS is stateless, what inc actually does is create a new Counter object with the incremented count variable.
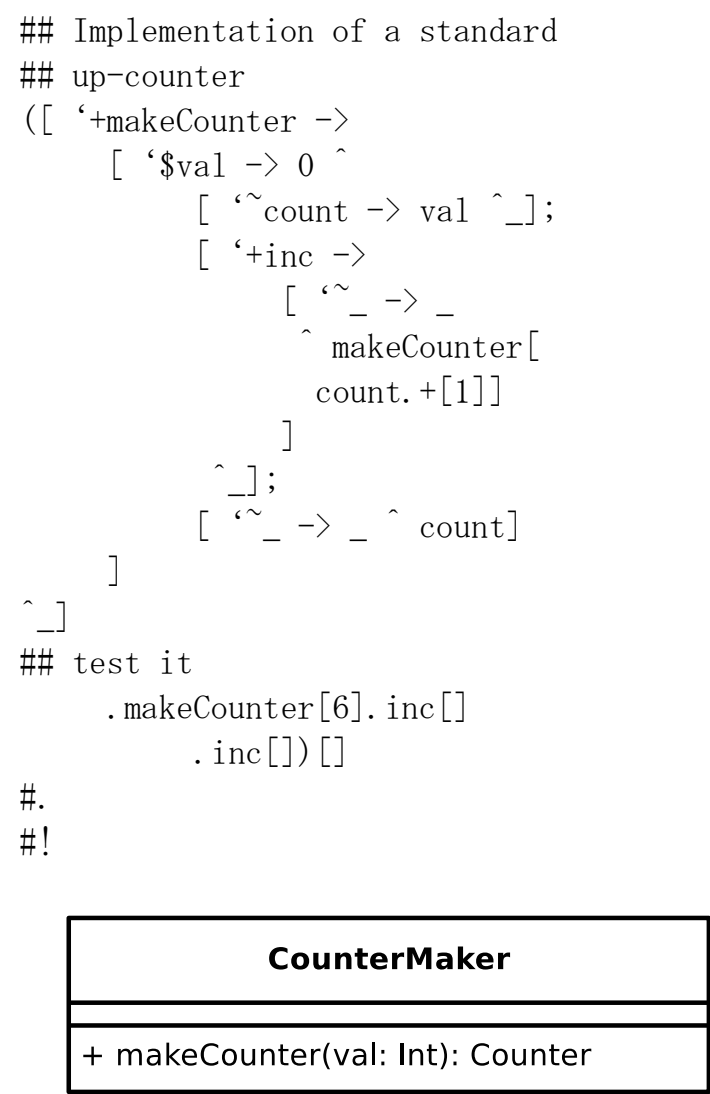

\begin{tabular}{|l|}
\hline Counter \\
\hline+ count: Int \\
\hline+ inc ()$:$ void \\
$+r v():$ Int
\end{tabular}

Fig.1. Class structure of Example (2)

Since UML is designed to model object-oriented systems, it is no surprise that using it to model a FOB requires extra notation to handle the ability to invoke a 
FOB as a function. In Fig. 1. the notation $r v$ is used to represent the operation of invoking the FOB as a function. The use of $r v$ (return value) in the diagram indicates that, when the FOB Counter is invoked, it returns the current value of the variable count.

Larger examples, and a more complete definition of the FOBS language are given by Gil de Lamadrid and Zimmerman ${ }^{[4]}$.

\section{CORE-FOBS DESIGN TOPICS}

Expression evaluation in FOBS-X is fairly straight forward. Three issues, however, need some clarification. These issues are: the semantics of the redefinition of a variable, the semantics of a FOB invocation, and the interaction between dynamic and static scoping.

\subsection{Variable overriding}

A FOB stack may contain several definitions of the same identifier, resulting in overriding. For example, in the following FOB

$$
\begin{aligned}
& \text { [ '\$m } \rightarrow \text { ' a' ‘ m.toInt }[]] \text {; } \\
& {\left[{ }^{\prime}+\mathrm{m}->3{ }^{\wedge} \mathrm{m}\right]}
\end{aligned}
$$

the variable $m$ has two definitions; in the super-FOB it is defined as an argument variable, and in the sub-FOB another definition is stacked on top with $m$ defined as a public variable. The consequence of stacking on a new variable definition is that it completely overrides any definition of the same variable already in the FOB stack, including the modifier. In addition, the new $\rho$-expression becomes the return value at the top of the full FOB stack.

\subsection{Argument substitution}

As mentioned earlier, the invoke operator creates bindings between formal and actual arguments, and then evaluates the $\rho$-expression of the FOB being invoked. At this point we give a more detailed description of the process.

Consider the following FOB that adds together two arguments, and is being invoked with values 10 and 6 .

$$
\begin{aligned}
& \left(\left[\$ \mathrm{r}->55_{-}\right] ;\right. \\
& \left.\left[\text {[ } \$ \mathrm{~s} \rightarrow 3{ }^{\wedge} \mathrm{r} .+[\mathrm{s}]\right]\right) \quad[10,6]
\end{aligned}
$$

The result of this invocation is the internal creation of the following FOB stack

$$
\begin{aligned}
& \text { [ } \left.\$ \mathrm{r}->5{ }^{\prime}\right] \text {; } \\
& {[\text { ' } \$ \mathrm{~s} \rightarrow 3 \wedge \text { r. }+[\mathrm{s}]] \text {; }} \\
& {['+r \rightarrow 6 \wedge r .+[\mathrm{s}]] \text {; }} \\
& {\left[{ }^{\prime}+\mathrm{s} \rightarrow 10 \wedge \text { r. }+[\mathrm{s}]\right]}
\end{aligned}
$$

In this new FOB the formal arguments are now public variables bound to the actual arguments, and the return value of the invoked FOB has been copied up to the top of the FOB stack. The return value of the original FOB can now be computed easily with this new FOB by doing a standard evaluation of its $\rho$-expression, yielding a value of 16 .

\subsection{Partial invocation}

Modern functional languages often support currying. The major contribution of currying is that it is a way to implement partial application, allowing the user to create a function from a function activation, with some, but not all of the parameters bound.

Originally FOBS implemented only the invoke operator, which combined argument binding and evaluation of the $\rho$-expression. Although the user could specify only a subset of the formal arguments, the result of a partial application was that the default values of the formal arguments would be used, and the return value would be a fully evaluated function, rather than a new partially applied function, as in currying.

When FOBS-X was developed from FOBS, a new operation, denoted as ",;", was added to the language. This operation, called partial invocation, implements a partial application in the semantic environment of FOBS. Although the mechanism in FOBS-X is radically different than currying, the result is close to the same.

The functioning of the partial invocation operator is best illustrated with an example. Consider the following example, using the invoke operator:

$$
\begin{aligned}
& \left(\left[\$ \mathrm{r}->55_{-}\right] ;\right. \\
& [\text {[S } \rightarrow 3 \text { 个. }+[\mathrm{s}]]) ; \text {; [10] }
\end{aligned}
$$

Here a FOB stack with two arguments, $r$, and $s$, is being invoked with only one actual argument; the value 10. When this happens, a new stack is formed, as discussed previously.

$$
\begin{aligned}
& \text { [ } \$ r->5 \text {, }] \text {; } \\
& {[\text { ' } \$ \mathrm{~s} \rightarrow 3 \text { r. }+[\mathrm{s}]] \text {; }} \\
& {\left[{ }^{\prime}+\mathrm{s} \rightarrow 10 \wedge \text { r. }+[\mathrm{s}]\right]}
\end{aligned}
$$

The $\rho$-expression of this stack, r. $+[\mathrm{s}]$ is then evaluated using the new binding for $s, 10$, and the default binding for $r, 5$, yielding the value 15 . This value is the value of the invoke operation.

Let us now consider the same example using the partial invocation operation.

$$
\begin{aligned}
& \left(\left[\$ \mathrm{r} \rightarrow 5 \mathrm{r}_{-}\right] ;\right. \\
& \text {[ '\$s } \rightarrow 3 \text { r. }+[\mathrm{s}]]) \text {; ; [10] }
\end{aligned}
$$

The partial invocation operator starts by performing argument binding, producing the same stack as the invoke operator. This is the stack of Example (3). However, unlike the invoke operator, there is no evaluation of the $\rho$-expression. The stack of Example (3) is the result of the partial invocation. This stack can later be supplied with more arguments, and fully invoked, as the user pleases. 


\subsection{Variable scope, and expression evaluation}

Scoping rules in FOBS-X are, by nature, more complex than scoping used in most functional languages. Newer functional languages, such as Haskell and ML, typically use lexical scoping. Dynamic scoping is often associated with older dialects of LISP.

Pure lexical scoping does not cope well with variable overriding, as understood in the object-oriented sense, which typically involves dynamic message binding. To address this issue, FOBS-X uses a hybrid scoping system which combines lexical and dynamic scoping. Consider the following FOB expression.

$$
\begin{aligned}
& \text { [ } \left.{ }^{\sim} \mathrm{y}->1^{\wedge}\right] \text {; } \\
& {\left[{ }^{\sim}{ }^{x}->\right.} \\
& \text { [ ' }+\mathrm{n}->\text { y. }+[\mathrm{m}] \text { ` } \mathrm{n}] \text {; } \\
& \text { [ ‘ } \left.{ }_{m} \rightarrow 2 \text { - }\right] \\
& \text {-] ; } \\
& {\left[{ }^{\sim}{ }_{\mathrm{Z}} \rightarrow 3 \text { ก } \mathrm{x} . \mathrm{n}\right]}
\end{aligned}
$$

We are currently mostly interested in the FOB stack structure of Expression (4), and can represent it graphically with the stack graph, given in Fig. 2. In the stack graph each node represents a simple FOB, and is labeled with the variable defined in the FOB. Three types of edges are used to connect nodes: the s-pointer, the tpointer, and the $\gamma$-pointer. The s-pointer describes the lexical nested block structure of one FOB defined inside of another. The s-pointer for each node points to the FOB in which it is defined. For example $m$ is defined inside of the FOB $x$.

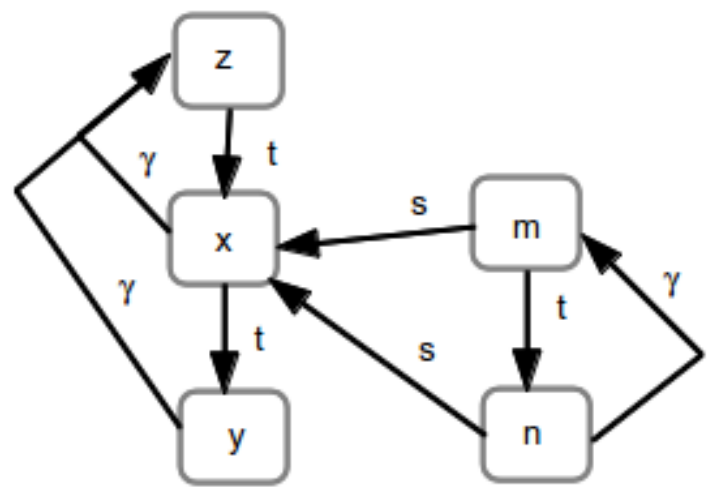

Fig.2. Stack graph of Example (4)

The t-pointer for each node points to the super-FOB of a FOB. It describes the FOB stack structure of the graph. In Fig. 2 there are basically two stacks: the top level stack consisting of nodes $z, x$, and $y$, and the nested stack consisting of nodes $m$, and $n$.

The $\gamma$-pointer is a back pointer, that points up the FOB stack to the top. This provides an easy efficient mechanism for finding the top of a stack from any of the nodes in the stack.

If the FOB $z$ were invoked, it would access the FOB $x$ for the value of $n$. This would cause the expression $y+m$ to be evaluated, a process that demonstrates the use of all three pointers. The process of resolving a reference in FOBS-X first examines the current FOB stack. The top of the current stack is reached by following the $\gamma$-pointer. Then the t-pointers are used to search the stack from top to bottom. If the reference is still unresolved, the spointer is used to find the FOB stack enclosing the current stack. This enclosing stack now becomes the current stack, and is now searched in the same fashion, from top to bottom, using the $\gamma$-pointer to find the top of the stack, and the t-pointers to descend to the bottom.

To illustrate this procedure for the example, to locate the definition of the variable $y$, referenced in the FOB $n$, the $\gamma$-pointer for $n$ is followed up to the FOB $m$, this FOB is examined, and then its t-pointer is followed down to the FOB $n$, which is also examined. Not having found a definition for the variable $y$, the s-pointer for FOB $n$ is followed out to the FOB $x$, and then the $\gamma$-pointer is followed up to the FOB $z$. FOB $z$ is examined, and its tpointer is traversed to FOB $x$, which is also examined. Then the t-pointer for FOB $x$ is finally followed down to the FOB $y$, which supplies the definition of $y$ needed in the FOB $n$.

As mentioned above, the scoping for FOBS-X is a combination of lexical and dynamic scoping. S-pointers are lexical in nature, since the nesting of FOBs is a static property. T-pointers and $\gamma$-pointers are dynamic. These pointers must be created as new FOB stacks are created during execution.

Table 1. Operations for the Boolean FOB

\begin{tabular}{|l|l|l|}
\hline Libary FOB & Operation & Description \\
\hline \hline Boolean & b.if $[\mathrm{x}, \mathrm{y}]$ & $\begin{array}{l}\text { If Boolean value } b \text { is } \\
\text { true, return } x, \\
\text { otherwise return } y\end{array}$ \\
\hline \hline b.\&[x] & $\begin{array}{l}\text { Return the boolean } \\
\text { value of the } \\
\text { expression } b \wedge x\end{array}$ \\
\hline \hline & b.|[x] & $\begin{array}{l}\text { Return the boolean } \\
\text { value of the } \\
\text { expression } b \vee x\end{array}$ \\
\hline \hline & b.![] & $\begin{array}{l}\text { Return the boolean } \\
\text { value of the } \\
\text { expression }\end{array}$ \\
\hline
\end{tabular}

\section{THE FOBS LIBRARY}

As FOBS-X can be extended by adding new primitive FOBs to the library, we use the term native primitive FOBs to denote the primitive FOBs that are part of coreFOBS. The FOBS library contains definitions of all native primitive FOBs. The native primitive FOBs are Int, Char, Real, Boolean, Vector, String, and FOBS. In addition a set of "mix-in" FOBs are contained in the library, that serve the same purpose as mix-in classes described by Page-Jones ${ }^{[9]}$, providing general capabilities to primitive FOBs. For example the Boolean FOB uses 
the mix-in FOBs $E q$, and Printable to supply operations to compare Boolean values for equality, and the ability to be printed, respectively.

The native primitive FOBs mostly implement the native data types of the FOBS language. Each data type provides the wrapper for the data, along with a set of operations, used to manipulate the data. As an example, Table 1 shows the operations provided by the Boolean FOB. This operation structure is shown in the UML diagram of Fig. 3. The operations for the Boolean FOB are implication, logical and, logical or, and logical not. The Boolean FOB inherits the operations of equals, and not-equals form the mix-in FOB $E q$, and it inherits the toString function, that generates a print-string, from the FOB Printable.

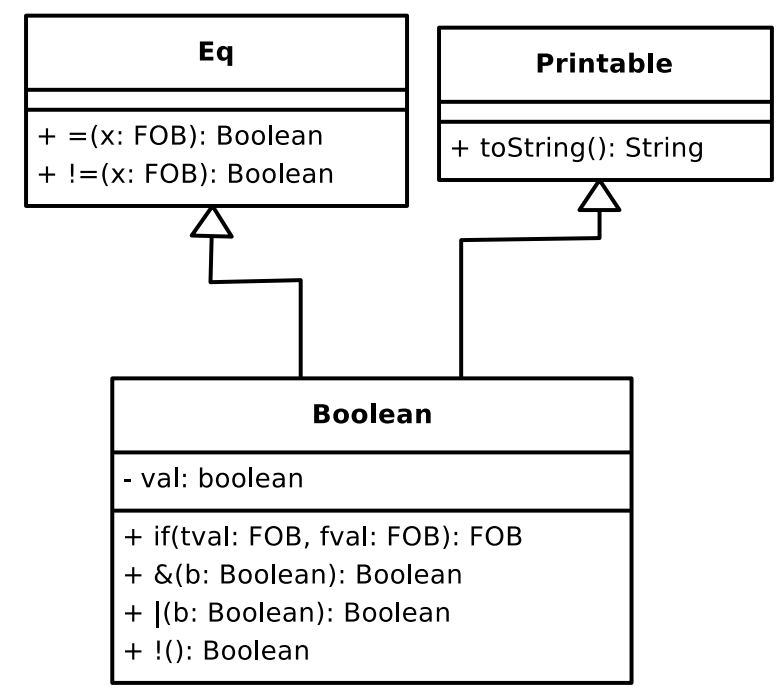

Fig.3. Interface for the Boolean FOB

The primitive FOB FOBS is the one primitive FOB that does not implement a data type. This FOB is, initially, largely empty. It, however, provides the mechanism for extending the FOBS-X language, allowing it to be adapted to differing scripting environments. The user of the FOBS-X language extends the language by adding modules to the FOBS FOB, one for each extension to the language.

\section{EXTENSIONS}

FOBS is a language that is designed to be extensible, both in terms of syntax, and semantics. To extend the language the user designs an extension. An extension is defined by an extension module, which is composed of two pieces: a macro file, and a collection of library modules.

\subsection{Macro files}

FOBS-X allows the syntax of the language to be changed in a limited fashion. The mechanism used to modify the syntax is macro expansion. Before a FOBS expression is parsed, a macro processor is used to expand macros used in the code. In this way, the user can alter the syntax of FOBS expression by writing and loading the appropriate macros to handle the changes.

Many programming languages have macro capabilities. These range from the fairly simple mechanisms in the programming language $C$, to the relatively more sophisticated mechanisms of LISP. It was felt that these simple systems were inadequate for FOBS. In particular, to implement a fair degree of flexibility, we felt that the ability to modify syntax should be more extensive than these types of systems offer, including a limited ability to change delimiter symbols. The language MetaML [13] provides much more sophisticated macro capabilities. It is built for the manipulation of macro type code, and implements multi-stage meta-programming. The macro capability of FOBS-X is much lighter weight than that of MetaML, but ideas from MetaML have found their way into FOBS-X. In particular, we found the staging of macro expansion useful. The staging in our case is used to implement macro operator precedence.

Macro definitions are quadruples, which are described in detail by Gil de Lamadrid ${ }^{[5]}$. Example (5) gives a simple demonstration of the form of macro definitions.

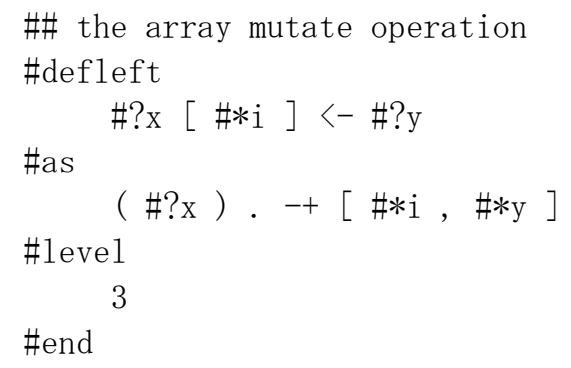

The macro quadruple consists of the following parts.

- $S_{1}$ : the search string, which includes wild-card tokens.

- $S_{2}$ : the replacement string, which includes wildcard tokens.

- $\quad P$ : the priority of the macro, with priority 19 being highest priority, and priority 0 being the lowest.

- $d$ : the direction of the scan, with right indicating right-to-left, and left indicating left-to-right.

In the FOBS notation of Example (5) the parts of the quadruple are specified using either the \#defleft, or the \#defright directive. Firstly, the directive specifies the direction $d$, depending on whether \#defleft or \#defright is used. Then the search string $S_{1}$, the replacement string $S_{2}$, and the priority $P$ are specified, in order, separated by the two delimiters \#as, and \#level, and terminated by the \#end directive.

The strings $S_{1}$, and $S_{2}$ are strings of FOBS lexicons, and wild-card tokens. Wild card tokens are all tokens that begin withe either the sequence "\#?" or "\#*", indicating a single wild card token, or a mutiple wild card token, respectively. A single wild card matches a single atom, and a multiple wild card matches a string of atoms. An 
atom is either a single FOBS token, or a balanced bracketed string, using one of the usual bracketing characters such as parentheses or braces.

Wild cards are named, so that the match in $S_{1}$ can be referred to in $S_{2}$. In Example (5), for example, the wild cards \#?x, \#?, and \#*i are matched in $S_{1}$, and their values are used in $S_{2}$.

The direction, $\mathrm{d}$, and the priority of a macro, $P$, are used to control the associativity of the operator defined by the macro, and the precedence of the operator, respectively. To control associativity, macros defined with direction left are expanded left-to-right, resulting in the definition of a left-associative operator, and macros defined with a direction of right are expanded right-toleft, resulting in a right-associative operator. To control precedence, macros with higher priority are expanded before macros with lower priority, resulting in operators with different precedences.

\subsection{The standard extension}

The syntax in core-FOBS-X is a little cumbersome. It has been designed with minimalistic notation, allowing a concise formal description, given by Gil de Lamadrid \& Zimmerman ${ }^{[4]}$. It is not necessarily attractive to the programmer. Standard extension (SE) FOBS-X attempts to rectify this situation. In particular, SE-FOBS-X includes constructs to enable the following.

- Allow infix notation for most operators.

- Eliminate the cumbersome syntax associated with declaring a FOB.

- Introduce English keywords to replace some of the more cryptic notation.

- Allow some parts of the syntax to be optionally omitted.

SE-FOBS-X is a language defined entirely using the macro processor. It demonstrates the flexibility of the FOBS-X macro capability to almost entirely rework the syntax of the language, without touching the back-end of the interpreter.

To help demonstrate the changes in syntax allowed by SE-FOBS-X, we rewrite the counter of Example (2) in SE-FOBS-X.

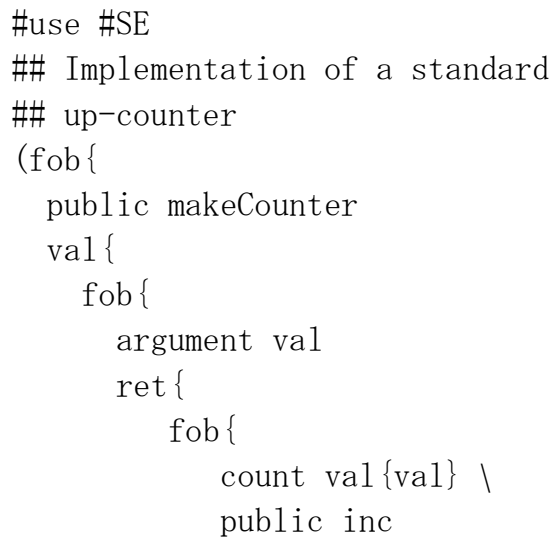

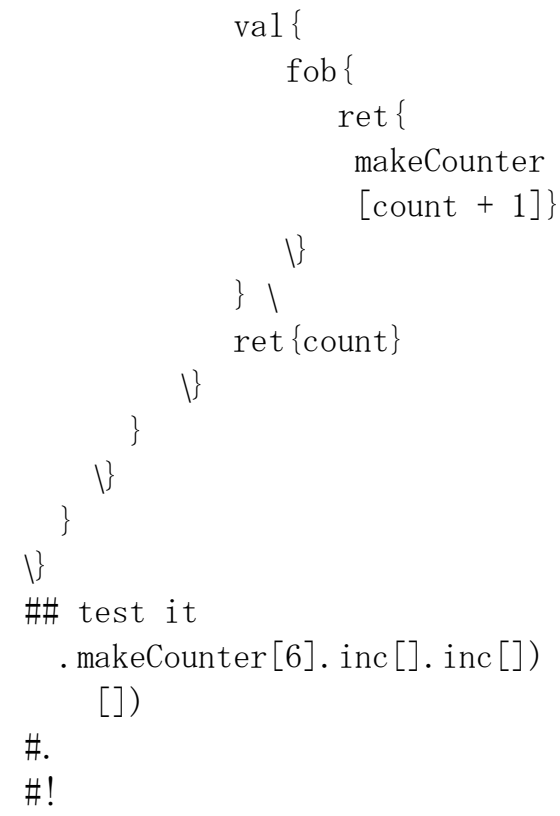

The \#use directive loads the standard extension macro file. This file makes available the syntax used in the remainder of the code. The most salient syntax feature of the code is the fob structure, used to define FOB-stacks. Each FOB in the stack is listed in the fob construct, and terminated by the " $\backslash$ " delimiter.

A FOB declaration contains a modifier, the identifier, a val structure, and a ret structure. The val structure defines the e-expression for the FOB, and the ret structure gives the $\rho$-expression for the FOB. Any of the parts of the FOB declaration may be omitted, resulting in the use of appropriate default values. Modifiers in SE-FOBS-X are the keywords public, private, and argument, instead of the cryptic symbols "“+", ", , and " \$".

A final feature present in Example (6) is the use of the infix version of the addition operator. All common binary operators in SE-FOBS-X are available in their infix version, relieving the user from using the normal coreFOBS access and invoke notation.

\subsection{Extension library modules}

Macro files extend the syntax of the FOBS language. To extend the semantics, you must add modules to the FOBS library. The FOBS-X library is written in Perl, and so to add modules you simply write Perl modules, and add them into the appropriate library directory structure. This process initially may sound simple. On further reflection, it becomes obvious that to do this

- One needs to be fairly familiar with the structure of the FOBS-X library.

- One must be familiar with how to manipulate FOBs in Perl.

While it is reasonable to expect a user requiring complex semantic changes to learn the required material to develop library modules from scratch, it is an unreasonable burden to impose on the user that desires to 
make only minor changes to the semantics of FOBS. To make small changes it is more appropriate for the user to do so using a tool that simplifies the process. The tool that we have developed is the FOBS Extension Definition Language Extension (FEDELE).

When designing FEDELE, we first thought of a metalanguage that was implemented as an external tool. However, since FOBS is a scripting language, and designed for just such work, we rapidly realized that it made sense to implement FEDELE as a FOBS-X extension. FEDELE is, therefore, a FOBS-X extension that helps the user create other FOBS-X extensions.

\section{THE FEDELE OPERATING ENVIRONMENT}

The standard extension is unusual in that it is an extension with only one component: the macro file. No semantic changes are made to FOBS; only syntactic changes. Most extensions contain both a macro file and library modules. FEDELE is a more usual extension. Library modules provide the capabilities of the package, and a macro file provides more convenient syntax for using it.

The FEDELE extension provides a simpler way of writing the library modules necessary for implementing an extension. The FEDELE language allows the user to specify the structure of the extension much in the same way that YACC (see Johnson ${ }^{[7]}$ ) allows a programming language designer to specify the structure of a new programming language. The specification is translated into a set of Perl modules implementing the extension. The modules are then placed in a directory, and the directory path is placed on the Perl include path @INC, extending the directories searched for library modules. This summarizes the process of extending the library, but to continue our discussion of FEDELE, we will need to examine the structure of the FOBS-X library in more detail.

\subsection{The FOBS library implementation}

The FOBS-X library is composed of a collection of primitive and utility FOBs. As explained previously primitive FOBs use utility FOBs to mix-in general capabilities. However, from the standpoint of structure, there is no difference between a primitive and a utility FOB. In this discussion we will therefor consider only the structure of a primitive FOB.

To illustrate the structure of a primitive FOB, we take as example the FOB Boolean. The Boolean FOB can be represented in UML as shown previously in Fig. 3. It contains an instance variable val that contains the actual Boolean value, represented as a character string. It also contains the common Boolean operations of and, "\&", or, "|", and not, "!". In addition it contains the operator if that implements the implication operator. The FOB Boolean inherits operations from the FOBs $E q$, and Printable. From $E q$ it inherits the operations equals, "=", and not-equals, "!=". From Printable it inherits the operation toString, that converts a Boolean value into a printable string.
It should be noted that the term "inheritance" for primitive FOBs is only loosely applied. In fact, the mechanism is more of a message-forwarding mechanism. That is to say that, for example, if a Boolean FOB receives an equals access request, the request is forwarded to its parent $E q$ FOB.

Implementing the Boolean FOB in Perl is done with two structures: a hash table, containing the data of the primitive FOB, and a Perl module, Boolean, that contains code for all of the operations in the primitive FOB.

\subsection{Primitive FOB Hash Table Structure}

The hash-table representing the data in a primitive FOB stores information in attribute-value pairs. The attributes of interest are the following.

- type - This attribute gives the type of the FOB. Using the notation described by Gil de Lamadrid \& Zimmerman ${ }^{[4]}$, a primitive FOB is of type "omega", and a non-primitive FOB has type "phi".

- code - This attribute stores the name of the primitive FOB. For the FOB Boolean, the code attribute would have the value "Boolean".

- Super-FOBs - This is a collection of attributes, one per parent FOB. Each of these attributes stores an instance of one of the parent FOBs. For the FOB Boolean there are two such attributes. superEq stores an instance of the primitive FOB $E q$, and superPrintable stores an instance of the primitive FOB Printable.

In addition to the above standard attributes, the primitive FOB hash-table contains attributes that are specific to the particular primitive FOB. For the Boolean primitive $\mathrm{FOB}$, there is only one more attribute: the attribute val, that holds the Boolean value of the FOB, stored as a character string.

\subsection{Primitive FOB Module Structure}

The main library module for a primitive FOB has the same name as the primitive FOB. For example, for the primitive FOB Boolean there is a Perl module called Boolean. This module has four standard functions in it.

- construct - This function constructs the hash table representing an instance of the primitive FOB.

- constructConstant - This function is an extension of the function construct. It constructs the instance, using construct, and then initializes it by filling in any instance variables.

- $\quad$ alpha - This is the function $\alpha$ that is described by Gil de Lamadrid \& Zimmerman ${ }^{[4]}$. It takes a single argument, a character string, and accesses the primitive FOB for the value of the identifier specified by the argument.

- $\quad$ iota - This is the function $\mathrm{l}$ described by Gil de Lamadrid \& Zimmerman ${ }^{[4]}$. It takes a single argument, a Vector FOB, and invokes the primitive FOB using the vector to supply its actual 
arguments.

\subsection{Operation Modules}

The main module of a primitive FOB is not the only module needed to define the FOB. To understand why this is so, consider the following FOBS code, and the semantics of invocation.

$$
\text { false. \& [true] }
$$

In this expression, the Boolean FOB false is being accessed for its and operation. The operation is then being invoked, with the argument true. However, the question arises, when we say that the operation is invoked, what, in fact, is an operation, in terms of implementation? The simple answer is that if an operation is invoked, then it must be a FOB, because only FOBs are invoked. This observation becomes trivially clear when we look at an example that does not involve a primitive FOB.

$$
\begin{aligned}
& {\left[`+\&->\left[{ }_{-} \rightarrow{ }_{-} \text {false }\right]\right.} \\
& \text { _]. \& [true] }
\end{aligned}
$$

In this example, as in Example (7), a FOB is accessed for an "\&" operation, and the operation is invoked with the Boolean FOB true. The difference is that in Example (8) the FOB being accessed is not a primitive FOB. What is produced by the access operation is a FOB, in this case, that always returns the value false. We observe that the same must be true of Example (7). That is to say that an access operation always produces a FOB, whether the FOB being accessed is a primitive FOB or not.

What the above discussion points out is that when we access an operator in a primitive FOB, what is produced is a FOB. That FOB, when invoked would perform the particular operation. Every operator in a primitive FOB must have defined a FOB that will perform the given operation. For a library FOB such as Boolean, each of its operators is defined as a primitive FOB. For example the and operator for the FOB Boolean is defined as a primitive library FOB called Boolean_and. We refer to library modules for the operations of a primitive FOB, as primitive operation modules.

To summarize, a primitive FOB is represented as a set of library modules. These consist of the main library module, described above, and a set of operation modules, one per operation. An operation module contains the same functions as the main module. That is to say that the operation module will have a construct function, a constructConstant function, an alpha function, and an iota function, each with the same role as in the main module. Each of these functions would perform actions appropriate to the operator. That is to say that the alpha function would always return an empty FOB, and the iota function would perform the operation of the operation module.

\subsection{Extension Access}

Once the user has defined an extension, the language FOBS-X must be able to allow the user to use the extension. This section describes the mechanism used to allow FOBS-X code to use an extension.

The modules of the extension can be placed at any location in the directory hierarchy of the operating system. The author of the extension then must inform the FOBS$\mathrm{X}$ interpreter where the extension is located. As discussed previously, this is done by ensuring that the extension directory is on the list of include directories for Perl, @INC. This is easily done by setting the environment variable PERL5LIB to the extension path.

Recall that the two components of an extension are the macro file, and the library modules. We discuss how the FOBS-X interpreter locates both of these components in this section. We begin with how the macro file is located. A macro file is loaded with the \#use directive. An example might be

\section{\#use Count}

This directive tells the FOBS-X interpreter to look for a file called Count.fobs containing the macros of the extension. What the FOBS-X interpreter does then is to search Perl include directories, listed in the array @INC. There are two exceptions to the procedure, as illustrated in the following \#use invocations.

$$
\begin{gathered}
\text { \#use \#SE } \\
\text { \#use \#FEDELE }
\end{gathered}
$$

The extensions \#SE, the standard extension, and \#FEDELE are considered part of the FOBS-X language, and as such are located in a separate default FOBS include directory.

We now turn to the location of library modules. The standard mechanism for accessing the library in FOBS is a reference to a constant. For example, if a FOBS expression contains a reference to the constant true, the FOBS interpreter observes that this is a Boolean constant. The interpreter then goes to the default library directory, locates the main Boolean module, and invokes its constructConstant constructor function to create the hashtable. ConstructConstant also links the main module to the hash-table, using a Perl mechanism called blessing, effectively making the hash table an object, in the objectoriented sense, which is to say that the hash table can be sent messages corresponding to any of the functions defined in the main Boolean module.

When the user defines their own library module, the above procedure cannot be used, because there is no FOBS constant for the new primitive FOB that would trigger the $\mathrm{FOB}$ construction. Instead, the construction of a FOB is triggered using the FOB FOBS. This is illustrated in the following FOBS expression. 


$$
\text { FOBS. Count. new [5] }
$$

The FOB FOBS is a primitive FOB in the FOBS-X library used to present links to extensions to the user. In Example (9), the user is attempting to access the identifier Count, which is the name of an extension. This identifier is not explicitly defined in the FOB FOBS. However, the FOBS-X interpreter will consider it implicitly defined, and, when referenced, will attempt to load the main module for the extension from the list of Perl include directories.

If we assume that the Count FOB is defined along the lines of the UML diagram in Fig. 1, the Count FOB has one operation, inc, explicitly defined. For every extension, generated by FEDELE or not, the primitive FOB must also contain a new operation. This operation, when called, generates a new instance of the FOB, and calls the constructConstant constructor for the FOB. In Example (9), the new operator is called to construct a primitive Count FOB, initialized to the value 5 .

\section{THE FEDELE EXTENSION}

This section describes the components of the FEDELE extension. FEDELE has both a macro file, extending the syntax of FOBS to more easily specify extension components, and library modules, providing the operators required to specify the contents of the library modules of the new extension, and write the module out. We begin by describing the FEDELE operations.

\subsection{The FEDELE Primitive FOB}

The primitive FOB FOBS.FEDELE is a very uncomplicated FOB that has no accessible identifiers in it, and can only be invoked. The result of an invocation is a FEDELE_module FOB. The FEDELE_module is a data structure used to collect information on the new FOB being described. Fig. 4 is the UML diagram showing the two FOBs: FEDELE, and FEDELE_module.

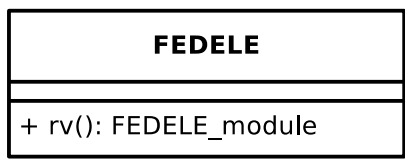

\begin{tabular}{|l|}
\multicolumn{1}{|c|}{ FEDELE_module } \\
\hline \begin{tabular}{l} 
- mixIn: String[0..*] \\
- makelD: String[0..*] \\
- element: StringPair[0..*] \\
- invokeValue: String[0..*] \\
- modulePath: String \\
\hline+ addMixIn(mi: String): FEDELE_module \\
+ addMakeld(mid: String): FEDELE_module \\
+ addElement(el: StringPair): FEDELE_module \\
+ addInvokeValue(iv: String): FEDELE_module \\
+ writeModule(path: String): FEDELE_module
\end{tabular} \\
\hline
\end{tabular}

Fig.4. Interface for FEDELE
The FEDELE_module FOB contains variables for storing the following items

- mixIn: a list of primitive mix-in FOBs.

- makeID: a list of identifiers that will be included in the hash-table representing the FOB.

- element: a list of operations that will be included in the FOB. Each operation is represented as a pair consisting of the operation name, and a snippet of Perl code that will become the body of the iota function for the operation.

- invokeValue: a snippet of Perl code that will become the body of the iota function for the new FOB itself.

- modulePath: the directory on to which the files of the library module will be written.

In addition to the above variables, the FEDELE_module also contains operators for adding items to its data structures. Each operation adds an item and returns the modified FEDELE_module.

\subsection{The FEDELE Macros}

The second part of the FEDELE extension is a macro file that defines the FEDELE language, and allows easier specification of a primitive FOB. The FEDELE language is a structured language. The structures of the language are listed in Table 2.

A FEDELE specification, at the outer level is an extension structure. This structure would contain clauses; each clause being either a mixIn, a make, an element, or an invoke structure. This is illustrated more clearly in the next section. FEDELE translates the extension structure into FOBS code that creates a FEDELE_module. The clause structures are translated into FEDELE_module operations that add the appropriate elements to the module. For example, the make clause would translate into an invocation of the addMakeId operator shown in Fig. 4.

\section{A FEDELE EXAMPLE}

We now present an example to illustrate how FEDELE is used. Suppose that the user wished to add a primitive FOB to the library that is similar to the counter FOB of Example (2). Remember that this example is illustrated in UML in Fig. 1. The new library FOB, however, unlike the counter of Example (2), will be mutable. That is to say that a counter will have state, and each time the counter is incremented it will change its state, rather than produce a new counter with the modified state. This new counter will also support two new syntactic constructs: one to easily construct a counter, and one to increment the counter. The syntax of these operations is illustrated in the following example.

$$
++(\% \mathrm{C}(5))
$$

This example uses the "\%C" operator to create a 
counter initialized to 5 . The second operator illustrated, "++", is used to increment a counter.

Table 2. FEDELE macro operations

\begin{tabular}{|c|c|}
\hline Structure & Description \\
\hline $\begin{array}{l}\text { extension " xtnd" } \\
\{\text { clauses }\} \text { to path }\end{array}$ & $\begin{array}{l}\text { Defines an extension with name } \\
x t n d \text {, to be written to the given } \\
\text { directory path. It contains clauses } \\
\text { giving the content of the } \\
\text { extension } x t n d \text {. }\end{array}$ \\
\hline $\operatorname{mix} I n$ mixin $F O B$ & $\begin{array}{l}\text { A clause indicating that the FOB } \\
\text { mixinFOB from the library is a } \\
\text { super-FOB for this FOB. This } \\
\text { clause can be repeated to include } \\
\text { several super-FOBs. }\end{array}$ \\
\hline make $\{$ idList $\}$ & $\begin{array}{l}\text { Describes the constant } \\
\text { constructor for the FOB. The } \\
\text { constructor will be available as } \\
\text { the function FOBS. } x \text { tnd. new. } \\
\text { New takes an argument for each } \\
\text { identifier listed, and stores the } \\
\text { argument as the value of the } \\
\text { identifier. The idList is given as a } \\
\text { list of strings, separated by } \\
\text { commas. }\end{array}$ \\
\hline $\begin{array}{l}\text { element "id" as } \\
\text { "perlScript" }\end{array}$ & $\begin{array}{l}\text { Gives the name of an element, or } \\
\text { operator, of the FOB available } \\
\text { through the access operator. The } \\
\text { included Perl script gives the } \\
\text { value returned if the operator is } \\
\text { invoked. }\end{array}$ \\
\hline invoke " perlScript" & $\begin{array}{l}\text { The Perl script gives the result of } \\
\text { an invoke operation on the FOB } \\
\text { itself. }\end{array}$ \\
\hline
\end{tabular}

Our new counter will also allow the user to increment the counter by any value, as opposed to just an increment of one. An increment of more than one will not be supported by the macros, but can still be accomplished by using the inc function itself, as in

$$
\text { c. inc }[3]
$$

that increases the value of the counter $c$ by 3 . Fig. 5 shows the new FOB structure in UML.

\subsection{The Counter FEDELE Specification}

The extension specification for our new counter consists of a FEDELE specification describing the library modules, and a macro file defining the syntax of the constructor operator, and the increment operator.

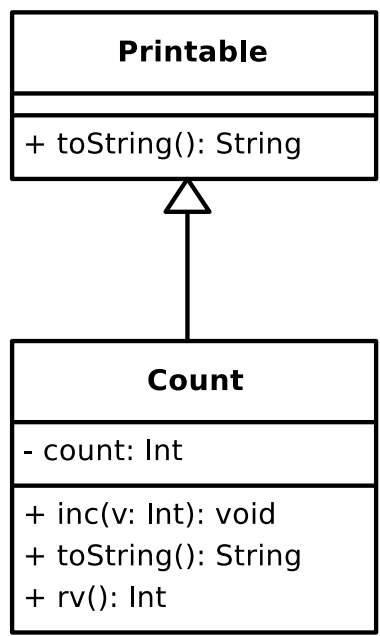

Fig.5. The mutable counter FOB

We begin by presenting the FEDELE code to generate the library modules.

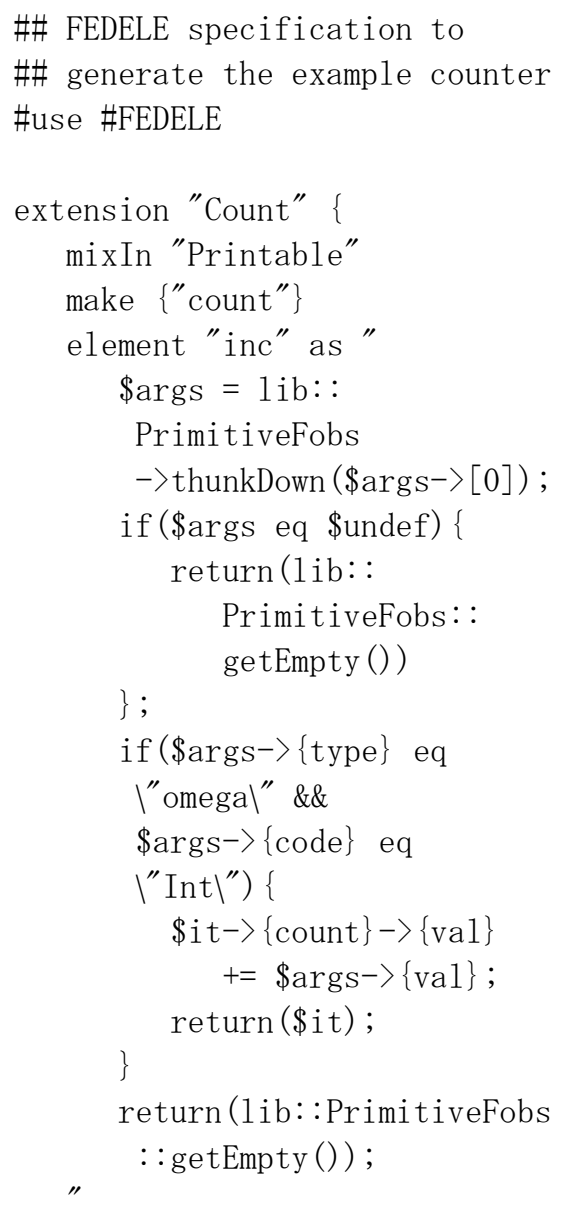




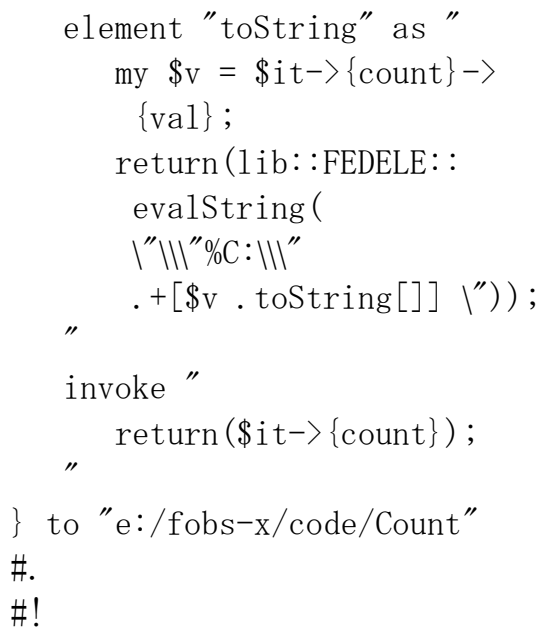

(10 cont.)

Considering the overall structure of Example (10), it is, in fact, faithful to the UML description of Figure 5. It specifies a mix-in FOB Printable, an identifier count, two operations, inc, and toString, and a return value when invoked.

The Perl code snippets from Example (10) illustrate several issues concerned with the interface between FOBS-X and Perl. The first issue is how to enable a Perl segment to access the arguments of the function call. This is accomplished through the use of several special variables.

- $\quad$ it - The FOB being operated on. That is to say that $\$$ it is the target of the invoke operation.

- $\quad$ \$args - A Vector FOB containing the arguments of the invoke operation.

The object $\$$ it contains all the identifiers declared in the FEDELE declaration as hash attributes. For instance, in the Count FOB, the sequence $\$ i t->\{$ count $\}$ is the count identifier of Fig. 5.

To access the arguments in the variable $\$ a r g$ a helper function is necessary. The arguments to FOBs are stored in thunks. To be used, the FOB inside the argument thunk must be unwrapped and evaluated. The function lib::PrimitiveFobs->thunkdown can be used for this purpose, as demonstrated in the definition of the operator inc.

There are a couple of other useful Perl functions used in Example (10). The function lib::PrimitiveFobs: :getEmpty can be used to create an instance of the empty FOB, a FOB often used to signal an exception. Another function lib::FEDELE: :evalString is used to evaluate FOBS expressions within the Perl code. This is a useful feature. Often it is easier to perform certain actions in FOBS, than in Perl. EvalString provides the ability to mix Perl with FOBS code, allowing the user to choose the more efficient implementation.

\subsection{The Counter Macro File}

The second component of the extension is the macro file that introduces more compact syntactic notation for the new counter operations. The contents of the file are shown in Example (11).

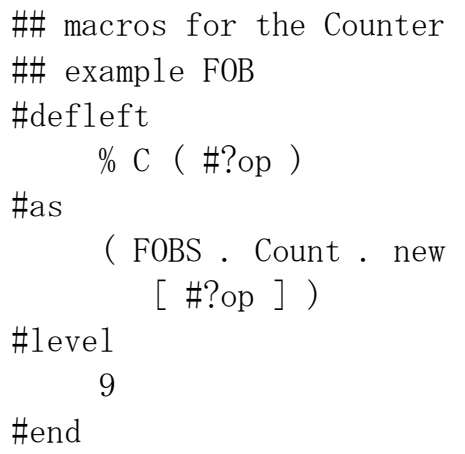

$$
\begin{aligned}
& \begin{array}{l}
\text { \#defleft } \\
++ \text { \#?op }
\end{array} \\
& \left.\begin{array}{l}
\text { \#as } \\
\text { \#?op . inc [ } 11
\end{array}\right] \\
& \text { \#level } \\
& \quad 8 \\
& \text { \#end } \\
& \text { \#! }
\end{aligned}
$$

The macro file contains the definitions of two macros. The first one implements the constructor structure with the "\% $\mathrm{C}$ " notation. It matches a string beginning with the character sequence "\% C", and followed by a single atom enclosed in parentheses. This sequence is replaced by an invocation of FOBS. Count. new with the matched atom as an argument.

The second macro is for the increment operator. It matches the operator " ++ " followed by an atom, and replaces it with an invocation of the inc operation on the matched atom with argument 1 .

\subsection{Stateful and stateless programing}

The counter defined by Examples (10) and (11) demonstrates rather graphically one of the issues concerning the hybrid paradigm of FOBS. There is a dichotomy between functional programming and objectoriented programming. The object-oriented paradigm clearly involves the explicit maintenance of state. In fact we often refer to the bindings of instance variables as the state of the object. On the other hand, although state does exist in functional languages, and is usually maintained by the system stack, it is not manipulated explicitly, in the sense that the program does not change the state directly as is the case in imperative and object-oriented programs, but rather indirectly by invoking functions. But, this difference between the two paradigms often becomes significant, and produces awkward situations in FOBS.

One of the defining characteristics of FOBS is referential transparency. This puts FOBS squarely in the camp of stateless programming. This is seen when we observe, for example, that identifiers can be bound to a value only once. Mutable objects are not an option in this style of programming. 
On the other hand, mutable objects are a staple of object-oriented programming. Also, state is often an integral part of scripting environments. For example, operating systems scripting often involves manipulating the state, represented as environment variables. To accommodate these situations in a language that advertises itself as an universal scripting language, it is not unreasonable for the user to wish to introduce state into FOBS. This is not difficult; the library can be extended to include mutable FOBs, as for example the Count FOB. However, it is still a stretch to use the language FOBS to manipulate these new mutable FOBs. In particular, what is needed to handle mutable FOBs is the ability to define operators whose return values are not used, but rather they are invoked only for their sideeffects on the state.

The problem of doing this type of stateful programming in a functional paradigm has been well researched, and has resulted in a body of literature on monadic programming (see Peyton Jones \& Wadler ${ }^{[10]}$, for example). Related to these results is a technique that has long been used in the object-oriented paradigm, called method chaining. This technique is used to pass multiple messages to the same object, as in the example

$$
\text { recipient. doX (xArg). doY (yArg) }
$$

in which the mutable object recipient is being first sent the message $d o X$ with the argument $x A r g$, followed by the message $d o Y$ with the argument $y A r g$. Although the operations $d o X$, and $d o Y$, naturally, might be thought of as returning no value, with the chaining technique they would instead return the object being operated on, recipient. In this way the next message in the chain is sent to the same recipient. One can think of the operators as passing the state along the chain from one operator to the next.

The technique of chaining is used in FOBS to handle mutable objects, allowing a sequence of operations resulting in state changes. Its effects can be observed in the code snippets of Example (10). The operator inc is defined to return the variable $\$ i t$, which is the target FOB, and this allows FOBS expressions such as $++(++\% \mathrm{C}(5))$, with a chain of increment operations being applied to the same FOB.

\section{FOBS AND SCRIPTING}

The intended use of FOBS-X is as a universal scripting language. Scripting languages are used to automate processes in a variety of environments. One of the most prevalent uses is in operating system interface. Scripting languages have also become very useful in creating dynamic web pages, and handling the collection of data using forms. They are also used in application programs, such as spreadsheets to automate calculations or procedures. In each of these applications the runtime system has two major components: an interpreter to execute scripts, and an interface that allows the script to interact with the environment. In FOBS-X, the library FOB FOBS is the interface to the environment. To adapt FOBS-X to a particular environment, an extension is created in the FOB FOBS. This extension contains all operations required for the interface, defined as FOBs.

As seen in the previous section, we have somewhat automated the process of creating these extensions. The user supplies a FEDELE description of an extension, and it is translated into a Perl definition.

We have commenced the construction of a UNIX extension. We present an example of how this extension might be used in scripting. A simple UNIX C-shell script follows that takes a command line argument, and prints out all file names in the current directory containing that string.

$$
\begin{aligned}
& \text { \#!/bin/csh } \\
& \text { if ( } \$ \text { \$argv }=0 \text { ) }) \text { then } \\
& \text { echo Usage: \$0 name } \\
& \text { exit } 1 \\
& \text { else } \\
& \text { set user_input }= \\
& \text { \$argv[1] } \\
& \text { 1s } \mid \text { grep i } \\
& \text { \$user_input } \\
& \text { endif } \\
& \text { exit } 0
\end{aligned}
$$

Assuming that an extension UNIX has been created, the above code could be translated into SE-FOBS-X as follows.

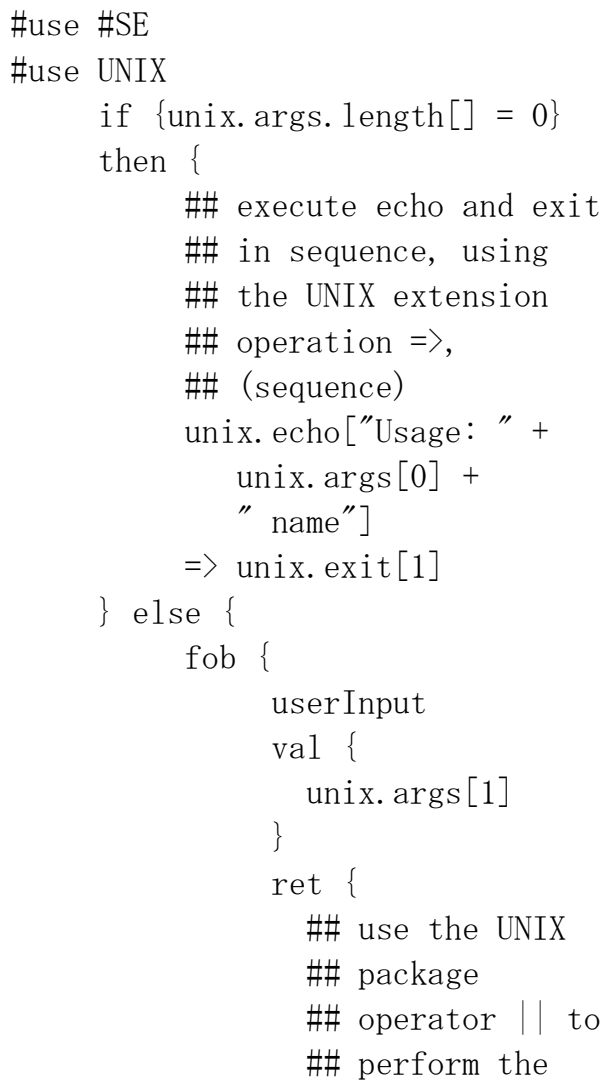




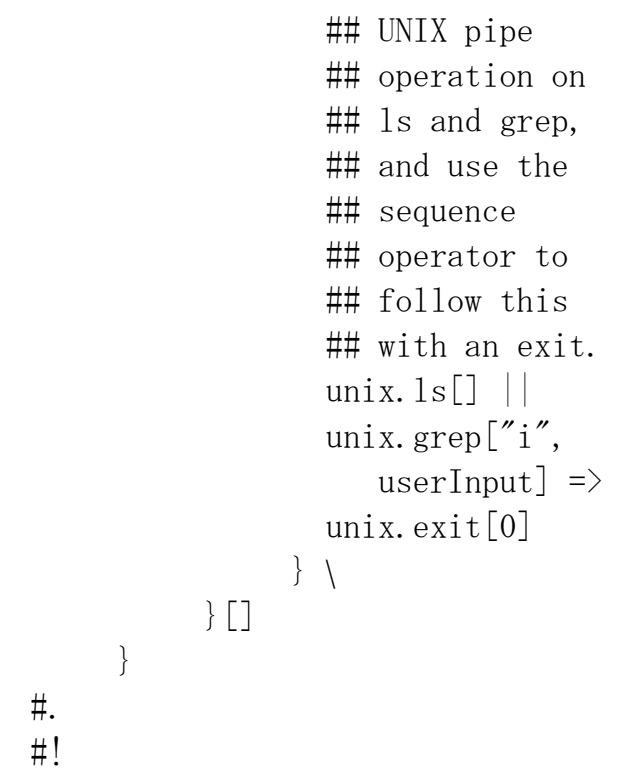

This script begins with two directives that inform the FOBS preprocessor that the standard and UNIX extensions are being used. The UNIX extension makes available the keyword unix, that is a convenience definition that allows the user to use this simple keyword, rather than the full specification, FOBS. UNIX.

Another notation defined in the UNIX extension is the operator "=> ", which might be called the sequence operation. This operator is used to interact with UNIX, which is stateful, using the FOBS computational model, which is stateless. In UNIX, operations are performed in sequence, and although they return values, they are usually performed for their side effects. The sequence operator takes as operands two FOBS expressions representing UNIX commands, performs them in sequence, alters the UNIX environment, and returns the return value of the last command as a FOB. The operator implements the chaining technique, discussed in Section 8.3.

A last notation used in the example is the operation "||". This is also part of the UNIX extension, and implements the UNIX pipe operation.

As a universal scripting language, FOBS-X will often be required to interact with stateful environments. The FOBS-X library gives FOBS-X that ability, although such interaction diminishes the referential transparency of the language. To ameliorate the situation, the library is structured to isolate all operations with side effects in the FOB FOBS.

\section{CONCLUSION}

We have briefly described a core FOBS-X language. This language is designed as the basis of a universal scripting language. It has a simple syntax and semantics.

FOBS- $X$ is a hybrid language, which combines the tools and features of object oriented languages with the tools and features of functional languages. In fact, the defining data structure of FOBS is a combination of an object and a function. The language provides the advantages of referential transparency, as well as the ability to easily build structures that encapsulate data and behavior. This provides the user with a choice of paradigms.

Core-FOBS-X is the core of an extended language, SEFOBS-X, in which programs are translated into the core by a macro processor. This allows for a language with syntactic sugar, that still has the simple semantics of our core-FOBS-X language.

Because of the ability to be extended, which is provided by SE-FOBS-X, the FOBS-X language gains the flexibility that enables it to be a universal scripting language. The language can be adapted syntactically, using the macro capability, to new scripting applications. The Extension FEDELE allows the semantics of the language to be adapted to new applications. FEDELE makes the process of extending the library easier, by automatically generating new library modules from a high-level specification language.

We are currently working on developing extensions for various scripting environments. Our next project is to produce a UNIX extension. Further in the future, we plan to investigate using FOBS for web scripting applications.

\section{REFERENCES}

[1] A. Alexandrescu The D Programming Language, Adison Wesley, 2010.

[2] M. Beaven, R. Stansifer, D. Wetlow, "A functional language with classes", Lecture Notices in Computer Science, vol. 507, Springer Verlag, 1991.

[3] D. Beazley, G. Van Rossum: Python; Essential Reference. New Riders Publishing, Thousand Oaks, CA. 1999.

[4] J. Gil de Lamadrid, J. Zimmerman, "Core FOBS: a hybrid functional and object-oriented language", Computer Languages, Systems \& Structures, vol. 38, 2012.

[5] J. Gil de Lamadrid, "Combining the functional and objectoriented paradigms in the FOBS-X scripting language", International Journal of Programming Languages and Applications, vol. 3, no. 2, AIRCC, Oct. 2013.

[6] J. A. Goguen, J. Mesegner, "Unifying functional, objectoriented, and relational programming with logical semantics", Research Directions in Object-Oriented Programming, pp. 417-478, MIT Press, 1987.

[7] S. C, Johnson, Yacc: Yet Another Compiler-Compiler. AT\&T Bell Laboratories. 2014.

[8] X. Leroy, D. Doligez, A. Frisch, J. Garrigue, D. Remy, J. Vouillon, The OCaml System Release 4.00: Documentation and Users Manual. Institut National de Recherche en Informatique et en Automatique, 2012.

[9] M. Page-Jones, Fundamentals of Object-Oriented Design in UML, pp. 327-336, Addison Wesley, 2000.

[10] S. L. Peyton Jones, P. Wadler, "Imperative functional programming", POPL, Charleston, Jan, 1993.

[11] S. S. Yau, X. Jia, D. H. Bae, "Proof: a parallel objectoriented functional computation model", Journal of Parallel Distributed Computing, vol. 12, 1991.

[12] M. Odersky, L. Spoon, B. Venners, Programming in Scala, Artima, Inc. 2008.

[13] T. Walid, T Sheard, "MetaML and multi-stage programming with explicit annotations", Proceedings of ACM SIGPLAN Symposium on Partial Evaluation and Semantic Based Program Manipulation, pp. 203-217, Amsterdam, NL, 1997. 


\section{Authors' Profiles}

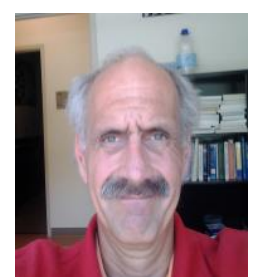

James Gil de Lamadrid: has a $\mathrm{PhD}$. in computer science, from the University of Minnesota, Minneapolis, Minnesota, USA. $\mathrm{He}$ is currently an Associate Professor at Bowie State University. He has multiple publications in the fields of robotics, and programming languages.

How to cite this paper: James Gil de Lamadrid,"Extending the Syntax and Semantics of the Hybrid Functional-ObjectOriented Scripting Language FOBS with FEDELE", International Journal of Information Technology and Computer Science(IJITCS), Vol.8, No.5, pp.13-27, 2016. DOI: 10.5815/ijitcs.2016.05.02 